\title{
Control-oriented Thermal Building Modelling
}

\author{
Sebastian Troitzsch ${ }^{1 *}$ and Thomas Hamacher ${ }^{2 \dagger}$ \\ ${ }^{1}$ TUMCREATE, Singapore, ${ }^{2}$ Technical University of Munich (TUM), Garching, Germany \\ Email: *sebastian.troitzsch@tum-create.edu.sg, ${ }^{\dagger}$ thomas.hamacher@tum.de
}

\begin{abstract}
Demand side flexibility (DSF) is becoming an important tool for the power system operation. With air-conditioned buildings being key candidates for DSF, this paper presents a control-oriented thermal building modelling approach which is kept in the linear domain and therefore suited for the employment in convex optimization problems. Along with the building model, two DSF indicators are proposed and applied to assess the DSF potential for an office building in Singapore.
\end{abstract}

\section{INTRODUCTION}

The integration of demand side flexibility (DSF) into the power system is an important step towards dealing with renewable generation and increased peak demand due to electric mobility. For example, DSF can help to match generation and demand by shifting flexible loads to time periods with high renewable generation [1], thereby avoiding the shedding of renewable generation and decreasing the need for additional energy storage systems [2]. Additionally, DSF can ensure that electric grid constraints, i.e., thermal limits and voltage limits, are maintained throughout the operation [3] or they can support the grid stability by offering reserves [4].

Heating, ventilation and air-conditioning (HVAC) systems are an important candidate for DSF as they account for a large share of the electricity demand in buildings. DSF from HVAC systems has seen increased attention with the advances in model predictive control (MPC) applications for buildings [5]. With MPC, the control problem of the HVAC system is expressed as a numerical optimization problem aimed at minimizing the operation cost, i.e., energy cost, while satisfying the occupant comfort constraints, i.e., acceptable limits for thermal comfort and indoor air quality. The building operator benefits from MPC through cost savings which arise from the ability to consider dynamic electricity tariffs [6], i.e., the electric demand is shifted to hours with low prices.

Appropriate mathematical building models are the key ingredient for MPC and thus for DSF. Specifically, the equations of the building model are constraints in the numerical optimization problem of the MPC. To this end, convex building model formulations are beneficial, because of the high computational efficiency of convex optimization solvers. This paper introduces a control-oriented thermal building model catering specifically to the formulation of MPC problems by keeping all model equation in the linear, i.e., convex, domain. This model expresses the relationship between the electric load of the HVAC systems and the indoor air climate with consideration for interactions of the building with its environment, its occupants and appliances. Compared to a similar modelling approach in [7], the present model proposes more detailed surface and HVAC model formulations which are better suited particularly for tropical climate. Furthermore, two DSF indicators are proposed and the model is employed to evaluate the DSF of an office building in Singapore.

\section{NOMENCLATURE}

Let $\mathbb{R}$ be the domain of real numbers. Non-bold letters $x, X$ denote scalars $\mathbb{R}^{1 \times 1}$, bold lowercase letters $\boldsymbol{x}$ denote vectors $\mathbb{R}^{n \times 1}$ and bold uppercase letters $\boldsymbol{X}$ denote matrices $\mathbb{R}^{n \times m}$. The transpose of a vector or matrix is denoted by ()$^{\top}$. Symbols for physical properties are aligned with ISO 80000 .

\section{BUILDING MODEL}

The thermal comfort is expressed in terms of the indoor air temperature. Hence, the thermal building model expresses the relationship between the indoor air temperature, the electric load of the HVAC system, the local weather conditions and the building occupancy. The indoor air temperature, i.e. zone temperature, within each zone is assumed to be uniformly distributed. As a starting point, the differential equation of the zone temperature $T_{z}$ of zone $z$ is expressed as:

$$
\frac{d T_{z}}{d t}=\frac{1}{C_{t h, z}} \cdot\left(\left(\sum_{s \in \mathcal{S}_{z}} \dot{Q}_{s, z}^{c n v, i n t}\right)+\dot{Q}_{z}^{i n f}+\dot{Q}_{z}^{o c c}+\dot{Q}_{z}^{h v a c}\right)
$$

Where $C_{z}^{t h}$ is the thermal heat capacity of zone $z \in \mathcal{Z}_{b}$, which is obtained according to ISO 13790 . The symbol $\mathcal{Z}_{b}$ is the set of all zones $z$ in building $b \in \mathcal{B}$ and $\mathcal{B}$ is the set of all buildings $b$. The heat transfer towards zone $z$ is composed of the the convective heat transfer $\dot{Q}_{s, z}^{c n v}$,int from surfaces $s \in \mathcal{S}_{z}$ towards zone $z$, heat transfer towards zone $z$ due to infiltration $\dot{Q}_{z}^{\text {inf }}$, heat transfer towards zone $z$ due to occupancy gains $\dot{Q}_{z}^{o c c}$ and heat transfer towards zone $z$ from the HVAC systems $\dot{Q}_{z}^{h v a c}$, where $\mathcal{S}_{z}$ is the set of all surfaces adjacent to zone $z$.

\section{A. Exterior surfaces}

Exterior surfaces are modelled as two thermal resistances with a centered heat capacitance between the exterior and zone $z$. Each surface $s$ is adjacent to exactly one zone $z$.

1) Heat balance: The heat balance for the exterior side of surface $s$ is expressed as:

$$
\dot{Q}_{s}^{c n v, e x t}+\dot{Q}_{s}^{i r r, e x t}-\dot{Q}_{s}^{e m s, s k y}-\dot{Q}_{s}^{e m s, g n d}=\dot{Q}_{s}^{c n d, e x t}
$$

Where $\dot{Q}_{s}^{c n v, e x t}$ is the convective heat transfer from the exterior towards surface $s, \dot{Q}_{s}^{i r r, e x t}$ is the incident irradiation onto surface $s, \dot{Q}_{s}^{e m s, s k y}$ and $\dot{Q}_{s}^{e m s, g n d}$ are the emitted radiation from surface $s$ towards the sky and the ground. Note that 
radiative heat exchange with the sky $\dot{Q}_{s}^{e m s, s k y}$ excludes any radiative heat exchange with the sun disc, which is modelled separately as irradiation $\dot{Q}_{s}^{i r r, e x t}$. The symbol $\dot{Q}_{s}^{c n d, e x t}$ describes the conductive heat transfer from the exterior towards the core of the surface.

The balance equation for the interior side of surface $s$ is expressed as:

$$
\dot{Q}_{s}^{c n d, i n t}=\dot{Q}_{s}^{c n v, i n t}-\dot{Q}_{s}^{i r r, i n t}
$$

On the interior side, $\dot{Q}_{s}^{c n d, i n t}$ is the conductive heat transfer from the core towards the interior side of the surface, $\dot{Q}_{s}^{c n v}$, int is the convective heat transfer from the interior side of surface $s$ towards zone $z$ and $\dot{Q}_{s}^{i r r, i n t}$ is the incident irradiation reaching surface $s$ through exterior windows adjacent to the same zone $z$.

The heat balance for the core of surface $s$ is expressed as:

$$
\frac{d T_{s}}{d t} C_{s}^{t h m}=\dot{Q}_{s}^{c n d, e x t}-\dot{Q}_{s}^{c n d, i n t}
$$

Where $C_{s}^{t h m}$ is the heat capacity of surface $s$. If the heat capacity of surface $s$ is neglectable $C_{s}^{t h m}=0$, e.g., for windows, the term simplifies to $\dot{Q}_{s}^{c n d, e x t}=\dot{Q}_{s}^{c n d, i n t}$.

2) Exterior convection: The exterior convective term $\dot{Q}_{s}^{c n v, e x t}$ is expressed as:

$$
\dot{Q}_{s}^{c n v, e x t}=A_{s} h^{c n v, e x t}\left(T^{a m b}-T_{s}^{e x t}\right)
$$

Where $A_{s}$ is the surface area of surface $s$ and $h^{\text {cnv,ext }}$ is the exterior convective heat transfer coefficient which is given according to ISO 6946 as $h^{c n v, e x t}=\left(0.04 \mathrm{~m}^{2} \mathrm{~K} / \mathrm{W}\right)^{-1}$. The symbol $T^{a m b}$ is the ambient temperature and $T_{s}^{e x t}$ is the temperature at the exterior side of surface $s$.

3) Exterior irradiation: The exterior irradiation term $\dot{Q}_{s}^{i r r, e x t}$ is expressed as:

$$
\dot{Q}_{s}^{i r r, e x t}=A_{s} \alpha_{s} \dot{q}_{d}^{i r r, e x t}, \quad d=d(s)
$$

Where $\alpha_{s}$ is the absorption coefficient of surface $s$ assuming a uniform absorption across the spectrum of the incident irradiation. The symbol $\dot{q}_{d}^{\text {irr, ext }}$ is the total incident irradiation onto a surface oriented towards direction $d=\{N, E, S, W, H\}$, i.e., vertically facing North $N$, East $E$, South $S$, West $W$ or horizontally facing upwards $H$, depending on the respective surface's orientation $d=d(s)$.

4) Exterior emissions: The exterior sky emission term $\dot{Q}_{s}^{e m s, s k y}$ describes the radiative heat loss through emission towards the sky. The term is expressed as:

$$
\dot{Q}_{s}^{e m s, s k y}=A_{s} h_{s}^{s k y}\left(T_{s}^{e x t}-T^{s k y}\right)
$$

In this linear approximation, the symbol $h_{s}^{s k y}$ is introduced as the sky heat transfer coefficient of surface $s$, whereas $T^{s k y}$ is the sky temperature. The sky heat transfer coefficient $h_{s}^{s k y}$ in turn is defined as:

$$
h_{s}^{s k y}=4 \sigma \varepsilon_{s} F_{s}^{s k y}\left(\frac{T_{s}^{s u r, e x t, l i n}+T^{\text {sky,lin }}}{2}\right)^{3}
$$

Where $\sigma, \varepsilon_{s}$ and $F_{s}^{s k y}$ are the Stefan-Boltzmann constant, the surface emission coefficient of surface $s$ for long-wave radiations and the view factor of surface $s$ towards the sky. The temperatures $T_{s}^{\text {sur,ext,lin }}$ and $T^{\text {sky,lin }}$ are linearization constants of $T_{s}^{e x t}$ and $T^{s k y}$. The term $F_{s}^{s k y}$ is defined as $F_{s}^{s k y}=0.5$ for $d(s) \in\{N, E, S, W\}$ and $F_{s}^{s k y}=1 d(s)=$ $H$, where $d(s)$ is the direction orientation of the surface.

The exterior ground emission term $\dot{Q}_{s}^{e m s, g n d}$ describes the radiative heat loss through emission towards the ground as well as the built environment. The term is expressed similar to $\dot{Q}_{s}^{e m s, s k y}$ as:

$$
\dot{Q}_{s}^{e m s, g n d}=A_{s} h_{s}^{g n d}\left(T_{s}^{e x t}-T^{a m b}\right)
$$

Where $h_{s}^{g n d}$ is introduced as the ground heat transfer coefficient of surface $s$, whereas $T^{a m b}$ is the ambient temperature. The ground heat transfer coefficient $h_{s}^{g n d}$ in turn is defined as:

$$
h_{s}^{g n d}=4 \sigma \varepsilon_{s} F_{s}^{g n d}\left(\frac{T_{s}^{s u r, e x t, l i n}+T^{\text {amb,lin }}}{2}\right)^{3}
$$

Where $F_{s}^{g n d}$ is the view factor of surface $s$ towards the ground. The temperatures $T_{s}^{\text {sur,ext,lin }}$ and $T^{a m b, l i n}$ are linearization constants of $T_{s}^{e x t}$ and $T^{a m b}$. The term $F_{s}^{g n d}$ is defined as $F_{s}^{g n d}=0.5$ for $d(s) \in\{N, E, S, W\}$ and $F_{s}^{g n d}=0 d(s)=$ $H$, where $d(s)$ is the direction orientation of the surface.

5) Interior convection: The interior convective term $\dot{Q}_{s}^{c n v, i n t}$ is expressed as:

$$
\dot{Q}_{s}^{c n v, i n t}=A_{s} h_{s}^{c n v, i n t}\left(T_{s}^{i n t}-T_{z}\right)
$$

Where $h_{s}^{c n v, i n t}, T_{z}$ and $T_{s}^{i n t}$ are the interior convective heat transfer coefficient, the zone air temperature and the temperature at the interior side of surface $s$. The interior heat transfer coefficient $h_{s}^{\text {cnv,int }}$ is defined according to ISO 6946 as $h_{s}^{\text {cnv,int }}=\left(0.13 \mathrm{~m}^{2} \mathrm{~K} / \mathrm{W}\right)^{-1}$ for $d(s) \in\{N, E, S, W\}$ and $h_{s}^{c n v, i n t}=\left(0.17 \mathrm{~m}^{2} \mathrm{~K} / \mathrm{W}\right)^{-1}$ for $d(s)=H$, where $d(s)$ is the direction orientation of the surface.

6) Interior irradiation: The interior irradiation term $\dot{Q}_{s}^{i r r, i n t}$ is expressed as:

$$
\dot{Q}_{s}^{i r r, i n t}=A_{s} \alpha_{s} \dot{q}_{z}^{i r r, i n t}, \quad d=d(s)
$$

Where $\dot{q}_{z}^{i r r, i n t}$ is the interior irradiation incident to all surfaces of zone $z$. The interior radiation $\dot{q}_{z}^{i r r, i n t}$ is in fact the irritation which has entered zone $z$ by passing through adjacent windows and is assumed to be uniformly distributed to all surfaces. This term is expressed as:

$$
\dot{q}_{z}^{i r r, i n t}=\frac{\sum_{w \in \mathcal{W}_{z}} A_{w} \tau_{w} \dot{q}_{d(w)}^{i r r, e x t}}{\sum_{s \in \mathcal{S}_{z}} A_{s}}
$$

Where $\tau_{w}$ is the transmission coefficient of window $w$. The sets $\mathcal{W}_{z}$ and $\mathcal{S}_{z}$ contain all windows $w$ and surfaces $s$ that are adjacent to zone $z$.

7) Conduction: Finally, the conductive terms $\dot{Q}_{s}^{c n d, e x t}$ and $\dot{Q}_{s}^{c n d, i n t}$ are defined:

$$
\begin{aligned}
& \dot{Q}_{s}^{c n d, e x t}=A_{s} 2 h_{s}^{c n d}\left(T_{s}^{e x t}-T_{s}\right) \\
& \dot{Q}_{s}^{c n d, i n t}=A_{s} 2 h_{s}^{c n d}\left(T_{s}-T_{s}^{i n t}\right)
\end{aligned}
$$

Where $h_{s}^{c n d}$ is the conductive heat transfer coefficient of surface $s$ and $T_{s}$ is the surface core temperature. For surfaces with 
a neglectable heat capacity, e.g., windows, the relationship simplifies to:

$$
\dot{Q}_{s}^{e x t, i n t}=A_{s} h_{s}^{c n d}\left(T_{s}^{e x t}-T_{s}^{i n t}\right)
$$

8) Complete model: Equations (2) to (14) and (15) define an overdetermined equation system, such that the temperatures $T_{s}^{e x t}$ and $T_{s}^{i n t}$ can be eliminated. However, the final equations which represent the complete model are omitted for brevity.

\section{B. Interior and adiabatic surfaces}

Interior surfaces are modelled as two thermal resistances with a centered heat capacitance between the zone $z_{1}$ and zone $z_{2}$. In principle, interior surfaces are modelled equivalently to exterior surfaces, where the heat balance for the interior side (eq. (3p) is applied for both sides of the surface. Adiabatic surfaces are modelled as a single thermal resistances between a heat capacitance and zone $z$. Adiabatic surfaces are modelled equivalently to exterior surfaces which are only the heat balance for the interior side (eq. (3)) is applied. For the sake of brevity, the full equations are omitted here.

\section{Infiltration}

The heat transfer towards zone $z$ due to infiltration $\dot{Q}_{z}^{\text {inf }}$ is defined as:

$$
\dot{Q}_{z}^{i n f}=V_{z} C^{t h, a i r} n_{z}^{i n f}\left(T^{a m b}-T_{z}\right)
$$

Where $V_{z}$ is the volume of zone $z, C^{t h, a i r}$ is the heat capacity of air and $n_{z}^{i n f}$ is the infiltration rate.

\section{Occupancy gains}

Assuming perfect knowledge of the building occupancy schedule, the heat transfer towards zone $z$ due to occupancy gains $\dot{Q}_{z}^{o c c}$, i.e., internal gains, is expressed as:

$$
\dot{Q}_{z}^{o c c}=A_{z} \dot{q}_{z}^{o c c}
$$

Where $A_{z}$ is the area of zone $z$ and $\dot{q}_{z}^{o c c}$ is the specific thermal gain due to occupancy.

\section{E. Heating, ventilation and air-conditioning (HVAC) systems}

HVAC systems are distinguished into 1) generic HVAC system, 2) air handling unit (AHU), 3) terminal units (TUs), 4) heating and chiller plant. The generic HVAC system (section II-E1 provides thermal heating / cooling power to each zone $z$, i.e. it directly adds / removes thermal energy to / from the zone. This generic HVAC system is an auxiliary system type which helps to model 1) simplified HVAC systems in case that a detailed model is not required or 2) HVAC system types for which a detailed model has yet to be implemented. The air handling unit (AHU) (section II-E2) serves supply air, i.e., conditioned outdoor air at a fixed temperature and humidity level, to each zone $z$. A terminal unit (TU) (section II-E3) serves supply air, i.e., re-conditioned zone air at a fixed temperature, to each zone $z$. Note that the TU takes in zone air, whereas the AHU draws fresh outdoor air. The heating and cooling demand of the AHU and TUs is provided in form of supply water, i.e., hot and chilled water, by the heating and chiller plant. The presented HVAC system models can in principal be amended for further system types, e.g., hydronic radiators, once appropriate linear models are formulated.

The total heat transfer towards zone $z$ from the HVAC systems $\dot{Q}_{z}^{h v a c}$ and the total electric demand of the HVAC systems $P^{\text {hvac,el }}$ is expressed as:

$$
\begin{aligned}
\dot{Q}_{z}^{h v a c} & =\dot{Q}_{z}^{g e n}+\dot{Q}_{z}^{a h u}+\dot{Q}_{z}^{t u} \\
P^{h v a c, e l} & =\sum_{z \in \mathcal{Z}}\left(\dot{P}_{z}^{g e n, e l}+P_{z}^{a h u, e l}+P_{z}^{t u, e l}\right)
\end{aligned}
$$

Where $\dot{Q}_{z}^{g e n}, \dot{Q}_{z}^{a h u}, \dot{Q}_{z}^{t u}, \dot{P}_{z}^{g e n, e l}, P_{z}^{a h u, e l}$ and $P_{z}^{t u, e l}$ are the heat transfer towards zone $z$ and electric power demand for the generic HVAC system, the AHU and the TU respectively.

1) Generic HVAC system: The heat transfer towards zone $z$ from the generic HVAC system $\dot{Q}_{z}^{g e n}$ is expressed as:

$$
\dot{Q}_{z}^{g e n}=\dot{Q}_{z}^{g e n, h e a t}+\dot{Q}_{z}^{g e n, c o o l}
$$

Where $\dot{Q}_{z}^{\text {gen,heat }}$ and $\dot{Q}_{z}^{\text {gen,cool }}$ are the thermal heating and cooling power provided to zone $z$. Note that $\dot{Q}_{z}^{\text {gen,heat }} \geq 0$ and $\dot{Q}_{z}^{g e n, c o o l} \leq 0$

The electric demand of the generic HVAC system is expressed as:

$$
\dot{P}_{z}^{\text {gen,el }}=\eta^{\text {gen,heat }} \dot{Q}_{z}^{\text {gen,heat }}+\eta^{\text {gen,cool }} \dot{Q}_{z}^{\text {gen,cool }}
$$

Where $\eta^{\text {gen,heat }}$ and $\eta^{\text {gen,cool }}$ are the efficiency factor for heating and cooling of the generic HVAC system and $P_{z}^{g e n, e l}$ is the electric power consumption of the generic HVAC system associated with thermal demand supplied at zone $z$. Note that $\eta^{\text {cool }}$ takes a negative value such that $P_{z}^{g e n, e l}$ is positive. The part-load behavior of the heating and chiller plant is neglected.

2) Air handling unit (AHU): The AHU model considers a cooling coil, a heating coil, supply air fan, exhaust air fan as well as a heat recovery system. For dehumidification, the air flow is assumed to be cooled below its dew point temperature and humidity is removed from the air by condensation at the cooling coil, then the air is reheated to supply air temperature. A humidifier is not considered in the model.

The heat transfer towards zone $z$ from the AHU $\dot{Q}_{z}^{a h u}$ is expressed as:

$$
\begin{aligned}
\dot{Q}_{z}^{a h u}= & \dot{V}_{z}^{a h u, h e a t} C^{\text {th }, \text { air }}\left(T^{a h u}-T_{z}^{\text {heat }, \text { lin }}\right) \\
& +\dot{V}_{z}^{a h u, c o o l} C^{\text {th,air }}\left(T^{a h u}-T_{z}^{c o o l, l i n}\right) \\
\dot{Q}_{z}^{a h u, h e a t}= & \left(\dot{V}_{z}^{a h u, h e a t}+\dot{V}_{z}^{a h u, c o o l}\right) \rho^{\text {air }} \\
& \cdot\left(\Delta h^{a h u, h e a t}-\Delta h^{a h u, h e a t, r e c}\right) \\
\dot{Q}_{z}^{a h u, c o o l}= & \left(\dot{V}_{z}^{a h u, h e a t}+\dot{V}_{z}^{a h u, c o o l}\right) \\
& \cdot \rho^{a i r}\left(\Delta h^{a h u, c o o l}-\Delta h^{a h u, c o o l, r e c}\right)
\end{aligned}
$$

Where $\dot{Q}_{z}^{a h u}$ is the heat transfer from the AHU towards zone $z, \dot{V}_{z}^{a h u, h e a t}$ and $\dot{V}_{z}^{a h u, c o o l}$ is the air flow rate for heating and cooling from the AHU towards zone $z$ and $C^{\text {th,air }}$ is the heat capacity of air. The symbols $T^{a h u}$ is the temperature of the conditioned supply air from the AHU and $T_{z}^{\text {lin,heat }}$ as well as $T_{z}^{l i n, c o o l}$ are the linearization constants of the air temperature 
at zone $z$ for heating and cooling. Further, $\dot{Q}_{z}^{a h u, h e a t}$ and $\dot{Q}_{z}^{a h u, c o o l}$ are the AHU heating and cooling demand to supply air for $z$ at set point conditions and $\Delta h^{a h u, h e a t}$ and $\Delta h^{a h u, c o o l}$ are the specific heating and cooling power applied to the outdoor air in order to obtain AHU supply air conditions. The symbols $\Delta h^{a h u, h e a t, r e c}$ and $\Delta h^{a h u, c o o l, r e c}$ are the specific heating and cooling power that is recovered from the return air. Note that the definition of $\Delta h^{a h u, c o o l, r e c}$ and $\Delta h^{a h u, h e a t, r e c}$ is omitted in the following for the sake of brevity. Heat gains due to the supply fan units are neglected in this model. The specific cooling and heating power are calculated such that the supply air is conditioned to the desired temperature set point $T^{a h u}$ and absolute humidity $x^{a h u}$. Depending on the ambient air temperature $T^{a m b}$ and ambient absolute humidity $x^{a m b}$, $\Delta h^{a h u, c o o l}$ and $\Delta h^{a h u, h e a t}$ are defined as:

$$
\begin{aligned}
& x^{a m, l} \leq x^{a h u}: \\
& \Delta h^{a h u, c o o l}=\min \left(0, h\left(T^{a h u}, x^{a m, l}\right)-h\left(T^{a m, l}, x^{a m, l}\right)\right) \\
& \Delta h^{a h u, h e a t}=\max \left(0, h\left(T^{a h u}, x^{a m, l}\right)-h\left(T^{a m, l}, x^{a m, l}\right)\right) \\
& x^{a m, l}>x^{a h u}: \\
& \Delta h^{a h u, c o o l}=h\left(x^{a h u}, \varphi=1\right)-h\left(T^{a m, l}, x^{a m, l}\right) \\
& \Delta h^{a h u, h e a t}=h\left(T^{a h u}, x^{a h u}\right)-h\left(x^{a h u}, \varphi=1\right)
\end{aligned}
$$

Where $T^{a m, l}$ and $x^{a m, l}$ are the linearization constants of the ambient air temperature and the ambient absolute humidity. The symbol $x^{a h u}$ is the absolute humidity at supply air setpoint conditions and is defined as $x^{a h u}=x\left(T^{a h u}, \varphi^{a h u}\right)$, where $x(T, \varphi)$ is the absolute humidity for a value pair of the dry-bulb temperature $T$ and relative humidity $\varphi$. The functions $h()$ and $x()$ give the specific enthalpy of wet air.

Finally, the the electric load due to the air supply to zone $z$ through the AHU $P_{z}^{a h u, e l}$ is expressed as:

$$
\begin{aligned}
P_{z}^{a h u, e l}= & \frac{1}{\eta^{\text {heat }}} \dot{Q}_{z}^{a h u, h e a t} \frac{1}{\eta^{\text {cool }}} \dot{Q}_{z}^{a h u, \text { cool }} \\
& +\dot{V}_{z}^{a h u} \Delta p^{a h u, f a n} \eta^{e l, a h u, f a n}
\end{aligned}
$$

Where $\eta^{\text {heat }}$ and $\eta^{\text {cool }}$ are the heating and cooling efficiency, i.e. the COP, of the heating and chiller plant. Note that $\eta^{\text {cool }}$ takes a negative value such that $P_{z}^{a h u, c o o l, e l}$ is positive. The symbols $\Delta p^{a h u, f a n}$ and $\eta^{e l, a h u, f a n}$ are the total pressure rise across all fans and the average electric efficiency coefficient of the fans.

3) Terminal unit $(T U)$ : The heat transfer towards zone $z$ from the TU $z \dot{Q}_{z}^{t u}$ is expressed as:

$$
\begin{aligned}
\dot{Q}_{z}^{t u} & =\dot{Q}_{z}^{t u, \text { heat }}+\dot{Q}_{z}^{t u, \text { cool }} \\
\dot{Q}_{z}^{t u, h e a t} & =\dot{V}_{z}^{t u, \text { heat }} C^{t h, \text { air }}\left(T^{t u, \text { heat }}-T_{z}^{\text {heat }, \text { lin }}\right) \\
\dot{Q}_{z}^{t u, \text { cool }} & =\dot{V}_{z}^{t u, \text { cool }} C^{t h, \text { air }}\left(T^{t u, \text { cool }}-T_{z}^{\text {cool,lin }}\right)
\end{aligned}
$$

Where $\dot{Q}_{z}^{t u, h e a t}$ and $\dot{Q}_{z}^{t u, c o o l}$ are the heat transfer for heating and cooling from the TU towards zone $z, \dot{V}_{z}^{t u, h e a t}$ and $\dot{V}_{z}^{t u, c o o l}$ are the air flow rate for heating and cooling from the TU towards zone $z$ and $C^{t h, a i r}$ is the heat capacity of air. The symbols $T^{t u, h e a t}$ and $T^{t u, c o o l}$ are the temperature of the conditioned supply for heating and cooling air from the TU and $T_{z}^{\text {heat,lin }}$ as well as $T_{z}^{\text {cool,lin }}$ are the the linearization constants of the air temperature at zone $z$ for heating and cooling.

Finally, the the electric load due to the air supply to zone $z$ through the TU $P_{z}^{t u, e l}$ is expressed as:

$$
P_{z}^{t u, e l}=\eta^{\text {heat }} \dot{Q}_{z}^{t u, h e a t}+\eta^{\text {cool }} \dot{Q}_{z}^{t u, c o o l}+\dot{V}_{z}^{t u} \Delta p^{t u, f} \eta^{e l, t u, f}
$$

Where $\eta^{\text {heat }}$ and $\eta^{\text {cool }}$ are the heating and cooling efficiency, i.e. the COP, of the heating and chiller plant. Note that $\eta^{\text {cool }}$ takes a negative value such that $P_{z}^{t u, c o o l, e l}$ is positive. The symbols $\Delta p^{t u, f}$ and $\eta^{e l, t u, f}$ are the pressure rise across the fan and the average electric efficiency coefficient of the fan.

\section{F. State space form}

The building model is transformed into state space form, because this allows for a more compact representation which is independent from changes in model configuration or parameters. The state space model is simply a vectorized representation of the differential equations for the zone temperature in eq. (1) and the surfaces in eq. (4), where the model variables are arranged into the following vectors:

$$
\begin{aligned}
\boldsymbol{x}= & {\left[\left[T_{z}\right]_{z \in \mathcal{Z}}^{\top},\left[T_{s}\right]_{s \in \mathcal{S}}^{\top}\right]^{\top} } \\
\boldsymbol{u}= & {\left[\left[\dot{Q}_{z}^{\text {gen }, \text { heat }}\right]_{z \in \mathcal{Z}}^{\top},\left[\dot{Q}_{z}^{\text {gen }, \text { cool }}\right]_{z \in \mathcal{Z}}^{\top},\left[\dot{V}_{z}^{\text {ahu,heat }}\right]_{z \in \mathcal{Z}}^{\top},\right.} \\
\boldsymbol{v}= & {\left.\left[\dot{V}_{z}^{\text {ahu }, \text { cool }}\right]_{z \in \mathcal{Z}}^{\top},\left[\dot{V}_{z}^{\text {tu,heat }}\right]_{z \in \mathcal{Z}}^{\top},\left[\dot{V}_{z}^{\text {tu,cool }}\right]_{z \in \mathcal{Z}}^{\top}, T^{\text {sky }},\left[\dot{q}_{d}^{\text {irr }}\right]_{d \in\{N, E, S, W, H\}}^{\top},\left[\dot{q}_{z}^{\text {occ }}\right]_{z \in \mathcal{Z}}^{\top}\right]^{\top} } \\
\boldsymbol{y}= & {\left[\left[T_{z}\right]_{z \in \mathcal{Z}}^{\top},\left[\dot{V}_{z}^{\text {ahu }}\right]_{z \in \mathcal{Z}}^{\top}, P^{h v a c, e l}\right]^{\top} }
\end{aligned}
$$

The vectors $\boldsymbol{x}, \boldsymbol{u}, \boldsymbol{v}, \boldsymbol{y}$ are the state, control, disturbance and output vectors. The time-discrete form of the thermal building model is obtained by application of zero-order hold discretization, which is omitted here for the sake of brevity. The final discrete-time state space model is expressed as:

$$
\begin{aligned}
\boldsymbol{x}_{t+1} & =\boldsymbol{A} \boldsymbol{x}_{t}+\boldsymbol{B}^{u} \boldsymbol{u}_{t}+\boldsymbol{B}^{v} \boldsymbol{v}_{t} \\
\boldsymbol{y}_{t} & =\boldsymbol{C} \boldsymbol{x}_{t}+\boldsymbol{D}^{u} \boldsymbol{u}_{t}+\boldsymbol{D}^{v} \boldsymbol{v}_{t}
\end{aligned}
$$

Where the matrices $\boldsymbol{A}, \boldsymbol{C}$ are the state and output matrix, and $\boldsymbol{B}^{u}, \boldsymbol{D}^{u}, \boldsymbol{B}^{v}, \boldsymbol{D}^{v}$ are the input and feed-through matrices, on the control and disturbance vectors respectively.

\section{DEMAND SIDE FLEXIBILITY (DSF) INDICATORS}

For evaluating the DSF of a particular building with the presented building model, two DSF indicators are proposed below. As a starting point, the optimal operation which will serve as the baseline for the DSF indicators is expressed as:

$$
\begin{array}{ll}
\min & \sum_{t \in \mathcal{T}} P_{t}^{\text {hvac,el }} c_{t} \Delta t \\
\text { s.t. } & 27 \quad \forall t \in \mathcal{T} \\
& T_{z, t}^{\text {min }} \leq T_{z, t} \leq T_{z, t}^{\max } \quad \forall z \in \mathcal{Z}, t \in \mathcal{T} \\
& \dot{V}_{z, t}^{\text {min }} \leq \dot{V}_{z, t}^{\text {ahu }} \quad \forall z \in \mathcal{Z}, t \in \mathcal{T}
\end{array}
$$


Where $T_{z, t}^{\min }, T_{z, t}^{\max }$ and $\dot{V}_{z, t}^{\min }$ are the minimum and maximum air temperature and minimum fresh air supply required at zone $z$, where ()$_{t}$ denotes the time-dependency of the constraints, e.g., to consider a night-setback. The symbols $c_{t}$ and $\Delta t$ are the electricity price at time step $t$ and $\Delta t$ is the time step length. For the following test case, the electricity price is set as $c_{t}=1$. The solution of eq. 28, i.e., $P_{t}^{\text {base }}=P_{t}^{h v a c, e l}$, is taken as the baseline electric load in the following.

\section{A. Maximum load reduction}

DSF can be characterized by the ability to defer electric load for a particular time period. To this end, the maximum load reduction for a fixed time period with respect to the baseline electric load is proposed as an indicator for DSF. The maximum load reduction factor $r^{\max }$ is determined with the following optimization problem:

$$
\begin{array}{ll}
\min & r_{t_{1}, t_{2}}^{\max }+\beta \sum_{\left\{t \in \mathcal{T} \mid t \notin\left[t_{1}, t_{2}\right]\right\}} P_{t}^{\text {hvac,el }} \\
\text { s.t. } & {[28 \mathrm{~b}), 28 \mathrm{c},(28 \mathrm{~d})} \\
& P_{t}^{\text {hvac,el }}=\left(1-r_{t_{1}, t_{2}}^{\text {max }}\right) P_{t}^{\text {base }} \quad t \in\left[t_{1}, t_{2}\right]
\end{array}
$$

Where $\left[t_{1}, t_{2}\right]$ is the desired load reduction time period and $\beta$ is a small weighting factor to ensure that the optimal solution considers demand minimization for time periods which are not in $\left[t_{1}, t_{2}\right]$. The optimization problem eq. (29) is iterated for different time period lengths $\Delta t=t_{2}-t_{1}$ and start times $t_{1}$ to obtain an average value for $r_{\Delta t}^{\max }$.

\section{B. Price sensitivity}

Assuming indirect control through energy prices, the building operator will determine its load schedule by minimizing the energy costs as in eq. (28). Therefore, the sensitivity of the electric load for changes in the price is proposed as an indicator for DSF. The load sensitivity $s_{\Delta c, t}$ for a change $\Delta c$ in the price $c_{t}$ at time step $t$ is expressed as:

$$
s_{\Delta c, t}=\frac{P_{t}^{\text {hvac }, e l}-P_{t}^{\text {base }}}{P_{t}^{\text {base }}}
$$

Where $P_{t}^{\text {hvac,el }}$ is the solution of eq. 28 for the updated $c_{t}$. The procedure is iterated over $t$ and $\Delta c$ to obtain an average value for $s_{\Delta c}$.

\section{RESULTS AND DISCUSSION}

The presented building model is applied for one storey of approx. $750 \mathrm{~m}^{2}$ at the CREATE Tower in Singapore, which is entirely occupied by office space. The test case building is equipped only with an AHU system, i.e., generic HVAC systems or TUs are not considered.

The maximum load reduction for the test case building is depicted in fig. 1. The largest load reduction is observed for a time period of $30 \mathrm{~min}$, while the possible load reduction decreases for longer time periods. This result demonstrates the limited thermal storage capabilities of the building.

The price sensitivity results are shown in fig. 2. The load variation takes positive values, i.e., the load increases, for price reductions and negative values, i.e. the load decreases, for price raises, which is consistent with the expected behavior.

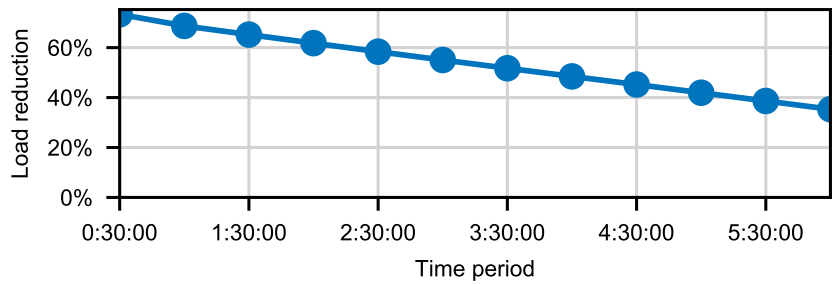

Fig. 1. Maximum load reduction.
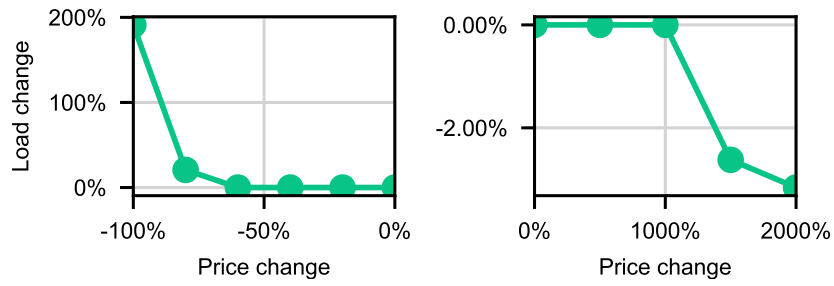

Fig. 2. Price sensitivity.

\section{CONCLUSION}

This paper introduced a control-oriented thermal building modelling approach for assessing the DSF of HVAC systems. Two DSF indicators where proposed and employed along with the building model to evaluate the DSF potential of a office in Singapore. The presented models, DSF indicators and test case are implemented in [8] and available open source 1$]$

\section{ACKNOWLEDGMENT}

This work was financially supported by the Singapore National Research Foundation under its Campus for Research Excellence And Technological Enterprise (CREATE) programme.

\section{REFERENCES}

[1] S. Hanif, C. Gruentgens, T. Massier, T. Hamacher, and T. Reindl, "Costoptimal operation for a flexible building with local PV in a Singaporean environment," in IEEE PES Innov. Smart Grid Technol. Conf., 2016.

[2] R. Atia and N. Yamada, "Sizing and Analysis of Renewable Energy and Battery Systems in Residential Microgrids," IEEE Trans. on Smart Grid, vol. 7, no. 3, pp. 1204-1213, 2016.

[3] O. Sundstrom and C. Binding, "Flexible Charging Optimization for Electric Vehicles Considering Distribution Grid Constraints," IEEE Trans. Smart Grid, vol. 3, no. 1, pp. 26-37, 2012.

[4] E. Vrettos, E. C. Kara, J. MacDonald, G. Andersson, and D. S. Callaway, "Experimental demonstration of frequency regulation by commercial buildings - Part I: Modeling and hierarchical control design," IEEE Trans. Smart Grid, 2016.

[5] F. Oldewurtel, A. Parisio, C. N. Jones, M. Morari, D. Gyalistras, M. Gwerder, V. Stauch, B. Lehmann, and K. Wirth, "Energy efficient building climate control using stochastic model predictive control and weather predictions," in Am. Control Conf., 2010.

[6] E. Vrettos, K. Lai, F. Oldewurtel, and G. Andersson, "Predictive Control of buildings for Demand Response with dynamic day-ahead and real-time prices," in Eur. Control Conf., Zurich, Switzerland, 2013.

[7] D. C. T. Sturzenegger, D. Gyalistras, V. Semeraro, M. Morari, and R. S. Smith, "BRCM MATLAB toolbox: Model generation for model predictive building control," in Am. Control Conf., 2014, pp. 1063-1069.

[8] S. Troitzsch, T. Miori, and A. Vautrin, "CoBMo - Control-oriented Building Model," Version 0.3.0, Zenodo, 2019. [Online]. Available: https://doi.org/10.5281/zenodo.3523539

${ }^{1}$ https://github.com/TUMCREATE-ESTL/cobmo 